\title{
Didáctica e inclusión en las aulas de matemática. Análisis de un caso en Chile
}

\author{
Didactics and Inclusion in Mathematics Classrooms. Analysis of a Case in Chile
}

\section{Didática e inclusão nas aulas de matemática. Análise de um caso no Chile}

Resumen: El artículo muestra, en dos apartados centrales, una temática educativa de gran relevancia vinculada a las necesidades educativas especiales y cómo estas han sido abordadas desde la incorporación de la educación especial al aula regular. La ruta de argumentos que van siendo conectados y que dan estructura a este texto expone fundamentos, conceptos, experiencias y reflexiones sobre un caso estudiado, con el objetivo de contrastar los principios teóricos y la realidad de aula, en relación con la educación inclusiva y las prácticas de enseñanza, para finalmente entregar sugerencias que favorezcan la renovación en los espacios de aprendizaje y la inclusión de cada estudiante en las clases de matemática.

Palabras claves: Diversidad; necesidades educativas especiales; dificultades de aprendizaje en matemática; inclusión.

Abstract: In this article, two central sections develop an educational theme of great relevance linked to special educational needs and how these have been addressed since the incorporation of special education into the regular classroom. The arguments being connected and structuring this text explain foundations, concepts, experiences, and reflections on a case studied. The aim is to contrast theoretical principles and the classroom's reality in relation to inclusive education and teaching practices, to finally give recommendations that favor the renewal of learning spaces and the inclusion of each student in math classes.

Keywords: Diversity; special educational needs; difficulties of learning in mathematics; inclusion. 
http://doi.org/10.15359/ree.25-1.23

http://www.una.ac.cr/educare

educare@una.ac.cr

\begin{abstract}
Resumo: O artigo mostra, por meio de duas seções centrais, um tema educacional de grande relevância vinculado às necessidades educacionais especiais e como estas vêm sendo abordadas desde a incorporação da educação especial à sala de aula regular. O percurso de argumentos que vão se articulando e que estruturam este texto expõe fundamentos, conceitos, experiências e reflexões sobre um caso estudado, com o objetivo de contrastar os princípios teóricos e a realidade da sala de aula, em relação à educação inclusiva e práticas de ensino, para finalmente entregar sugestões que favoreçam a renovação dos espaços de aprendizagem e a inclusão de cada aluno nas aulas de matemática.
\end{abstract}

Palavras-chave: Diversidade; necessidades educativas especiais; dificuldades de aprendizagem em matemática, inclusão.

\title{
Introducción
}

El siguiente ensayo adopta características de índole teórico y práctico para exponer la temática relativa a las necesidades educativas especiales (NEE), específicamente en matemáticas, en el aula común y los principios que rigen su contención con los parámetros actuales vinculados a la educación inclusiva.

El primer apartado plantea y sintetiza antecedentes que sostienen la temática de las dificultades de aprendizaje en matemáticas, su estado del arte y cómo estas constituyen una realidad recurrente en las salas de clases. El tema de las NEE, su diagnóstico integral y mediación parece demostrar aún falencias en los métodos de aula, que no logran contener adecuadamente la diversidad aparente y consustancial a cualquier contexto pedagógico. Esta es atribuida generalmente a este segmento de estudiantes y no es más que un estado inherente a la condición natural de las personas, pero que permanece subyugada a los intentos de control y homogenización que inclusive en el ámbito educativo permanecen. Sin embargo, desde la mirada de la educación inclusiva como un derecho, educar es ofrecer las mismas posibilidades de participación y aprendizaje (United Nations Eductional, Scientificand Cultural Organization [UNESCO], 2005) a todo el estudiantado, independientemente de sus características individuales, etnia, lengua, discapacidad o necesidades excepcionales de aprendizaje. Desde este enfoque, la inclusión se orienta hacia los derechos educativos de "aquellos grupos de alumnos que podrían estar en riesgo de marginalización, exclusión, o fracaso escolar" (Echeita Sarrionandía y Ainscow, 2011, p. 34), en el cual las escuelas deben visualizar la diferencia como una opción para prosperar el aprendizaje en el aula (UNESCO 2005; Winter y O'Rawl, 2010).

El segundo apartado se esmera en sintetizar los antecedentes de un estudio realizado en una escuela municipal de la ciudad de Talca, con el afán de exponer, analizar e interpretar los elementos didácticos y matemáticos en entornos pedagógicos vulnerables, de notoria heterogeneidad, con respecto a las NEE. Desde la perspectiva holística de la Didáctica de la Matemática, que aporta una visión que concibe la matemática como una estrategia desarrollada 
por el ser humano a lo largo de su historia para explicar, entender y convivir con la realidad dentro de un contexto natural y cultural (D'Ámbrósio, 2005), con lo cual la formación en este ámbito es entendida como un aspecto integral en la vida de las personas y debe estar al alcance de todo el alumnado por medio del "acceso a ideas matemáticas relevantes, cómo pensar de manera efectiva con estas ideas, y cómo aplicar sus conocimientos matemáticos más allá de los muros de la clase" (Diaz Godino, 2005, p. 8).

Ambos apartados han sido extraídos de procesos investigativos en el marco de las exigencias para un programa de Doctorado y Magister en Didáctica de la Matemática de la Universidad Católica del Maule, y buscan, de cierta forma, transparentar la necesidad de aportar, con fundamentos, desde la teoría didáctica, a un fenómeno de estudio que, aunque habitual y a veces anónimo es casi invisibilizado para el sistema educativo.

En este artículo se revelan argumentos y antecedentes de una experiencia investigativa que, de manera natural, tienden a ser comparados. El primero muestra una radiografía de la actualidad con respecto a este tema y el segundo lo que sucedía hace unos cinco años. Lo cierto es que parecen coincidir en instantes del relato como si estuvieran conectados.

Los antecedentes del caso estudiado son interpretados desde la óptica de los criterios de idoneidad didáctica delimitados en el enfoque ontosemiótico, que permiten examinar las prácticas de clases e instrucción, concatenación y logro de las tareas de aprendizaje, evaluación de capacidades matemáticas del alumnado, así como también, factores e interacciones que interceden en estos procesos (Diaz Godino et al. 2006).

Las consideraciones finales son desarrolladas a modo de reflexión en primera persona, pues sin haber sido la intención, al inicio del desarrollo de este texto, se decide dejar este estilo de escritura, ya que, por medio de este segmento del escrito, se pretende transmitir lo fundamental de remirar el tema expuesto y las implicancias didácticas tanto para la investigación educativa como para la implementación de metodologías de enseñanza en la clase de matemática.

\section{Dificultades de aprendizaje en matemáticas en contextos de inclusión. Reflexión y argumentos}

La matemática constituye un ámbito del saber en el que su enseñanza y aprendizaje, sigue siendo un motivo de atención. Pues, como plantea Duval (2016), adquirir este conocimiento involucra procesos complejos e investigar sobre cómo estos se desarrollan, implica desafíos desde lo teórico y lo educativo.

Los antecedentes reunidos por Maher et al. (2018), en los últimos estudios internacionales sobre la enseñanza matemática para niveles primario y elemental, transparentan que, aunque se avanza en las construcciones teóricas sobre el rol docente y su influencia en el desarrollo 
http://doi.org/10.15359/ree.25-1.23

http://www.una.ac.cr/educare

educare@una.ac.cr

del entendimiento y razonamiento matemático, estas convergen en delinear conceptualmente enfoques de instrucción, que aún visualizan al alumnado en entornos uniformes de aprendizaje, en los que el estudiantado con logros académicos esperados para su nivel sigue siendo la prioridad. Sin embargo, la realidad del aula, y específicamente de la clase de matemática, expone estratos y contextos de aprendizaje diferentes, en los que existe un fragmento de estudiantes que se desarticula tanto de la clase y aprendizaje imperante, como de los métodos de enseñanza habituales (Jimeno, 2006). Esta disociación, ocurre en la manifestación de condiciones excepcionales de aprendizaje -estimadas en aritmética principalmente- que confluyen en lo que se reconoce hoy, como dificultades de aprendizaje en matemáticas (Aguilar Villagrán et al., 2015), que para Kaufmann et al. (2013) corresponden a un variado espectro de trastornos asociados a diferentes causas y factores etiológicos, como déficits en el funcionamiento numérico a nivel cognitivo y disfunciones de procedencia no numérica. Discalculias o acalculias son parte de las primeras expresiones de índole clínico, que surgen de aludir a estas nociones y a las que distintos estudios, ya en la década de los 60, como los de Hécaen et al. (1961) y otros, las categorizan para explicar falencias en el desarrollo de destrezas y procedimientos aritméticos, cálculos numéricos, comprensión de la cantidad, adquisición de funciones básicas, ejecutivas y nociones elementales. Aguilar Villagrán et al. (2015) mencionan que las diversas expresiones y términos son utilizados para denominar hechos similares. Barallobres (2016) las declara en relación con las interpretaciones que trascienden de las diferentes corrientes teóricas, en las cuales las ciencias cognitivas aparecen como su principal referencia de análisis.

En nuestro país, las normativas vigentes las delimitan en el ámbito de las necesidades educativas especiales (NEE) de carácter transitorio y los trastornos específicos de aprendizaje (TEA).

Párrafo 10

Del Trastorno Específico del Aprendizaje.

Artículo 23.- Se entenderá por Trastorno Específico del Aprendizaje, en adelante dificultades específicas del aprendizaje, a una dificultad severa o significativamente mayor a la que presenta la generalidad de estudiantes de la misma edad, para aprender a leer; a leer y a escribir; y/o aprender matemáticas. (Ministerio de Educaciónde Chile [MINEDUC], 2010, p. 8)

En matemática, si no se explican por una discapacidad intelectual o escolarización inadecuada, los criterios de diagnóstico se establecen a partir de la adquisición de conocimientos básicos de operatoria, concepto de número o resolución de problemas prenuméricos. Criterios un tanto limitados y que aparentemente hacen referencia a diagnósticos en etapa temprana de escolarización, por los conceptos matemáticos considerados en su definición y que parece fusionar dos términos (dificultad y trastorno), los cuales no implican los mismos impedimentos 
http://doi.org/10.15359/ree.25-1.23

para aprender, ni las mismas causas o sugerencias de reeducación. De acuerdo con Lombardino (2017), la dificultad se define en relación con el rendimiento esperado para un nivel de enseñanza, en referencia a evaluaciones estándar y el trastorno, a la presencia de un déficit en comorbilidad, "pueden parcialmente coincidir, sobre todo cuando el trastorno es de nivel ligero; o cuando no se pueden recoger los informes necesarios para individuar el problema; pero, además, cuando la individuación del problema escolar no es validada por una diagnosis clínica" (p. 22). Las formas de detectar estas problemáticas de aprendizaje y las consecuencias de situar al alumnado en una categorización determinan, finalmente, estrategias de abordaje e intervención pedagógica en ocasiones inadecuadas, que la mayoría de las veces le acompañan durante toda su etapa escolar. Estudios en Europa e Italia, específicamente, demuestran que "el fenómeno del etiquetado en una categoría diagnóstica de los estudiantes que tienen un bajo rendimiento en matemática ha creado confusión respecto a que se entiende por dificultad o trastorno (discalculia)" (González et al., 2017). Ello, según estos autores, influye en la totalidad de miembros al interior de una comunidad escolar, ya que los cuerpos docentes no poseen la suficiente preparación o apoyos necesarios para dar respuesta a esta desemejanza de necesidades educativas en la clase de matemática, quizás, hasta erróneamente evaluadas.

En Chile, una vez diagnosticado el alumnado que presenta tal clase de NEE, a menudo, es incorporado, de acuerdo con las normativas vigentes, a los programas de integración escolar considerados como "una estrategia de apoyo inclusivo" (MINEDUC, 2019), lo que debiera significar un despliegue de medidas de apoyo adicionales acordes con sus requerimientos individuales de aprendizaje, como, por ejemplo, el trabajo interdisciplinario, la enseñanza adaptada y flexible o recursos específicos. No obstante, antecedentes como los expuestos por Tenorio (2011) indican que el personal docente chileno adjudica una connotación negativa a los procesos de integración escolar, ya que experimenta obstáculos al implementar estas políticas y, a la vez, para favorecer la mejora académica de la niñez integrada. Tenorio (2011), además, concluye sobre la urgencia de fortalecer e innovar en la formación del profesorado sobre temáticas relativas a las NEE y al trabajo pedagógico para responder a estas demandas educativas.

Los estándares pedagógicos de formación y egreso del profesorado en educación básica, en estos ámbitos (MINEDUC, 2011) resumen ciertos parámetros de competencias profesionales que consideran el respeto y valoración de la diversidad en el aula, conocimiento de métodos y dispositivos didácticos pertinentes para abordarla como la adaptación de la enseñanza y de acceso al currículo, de las normativas que rigen la integración escolar de estudiantes y los fundamentos que les posibilitan seguir procedimientos de derivación y trabajo interdisciplinario (MINEDUC, 2011). De forma paralela, los estándares disciplinares específicos en matemática consideran dos áreas fundamentales: el saber la disciplina para enseñar y saber enseñar la disciplina, que, subdivididas por eje temático, contemplan la identificación de errores o 
http://doi.org/10.15359/ree.25-1.23

http://www.una.ac.cr/educare

educare@una.ac.cr

dificultades en el aprendizaje, por cada contenido disciplinar. Sin embargo, y a pesar de que esto será parte de las circunstancias complejas de desempeño a las que se enfrentará el futuro profesorado (lo que no es desconocido para nadie), los referentes contenidos globalmente en estos estándares, y que delimitan las directrices orientadoras para las instituciones de educación superior, no explicitan claramente el dominio profundo o detallado de prácticas específicas de detección o cómo abordarlas metodológica y colaborativamente en el aula.

Infante et al. (2008 en Infante, 2010) afirman que las competencias para un futuro profesor o profesora "no necesariamente son aquellas que el mismo estudiante en formación ha elaborado a través de sus experiencias en los establecimientos educacionales" (p. 292).

Para esta misma autora (Infante, 2010) el hecho de que los procesos formativos determinen ciertas capacidades profesionales y estas no coincidan con las que va necesitando en los sucesivos episodios de formación práctica, también es visualizado en futuros grupos de profesionales especialistas como el profesorado de educación diferencial o especial. Sus perfiles formativos y de egreso, además de confrontarse entre sí, lo hacen con la realidad educativa, pues estos consideran dos visiones distintas del alumnado en la sala de clases: una predominantemente homogénea y colectiva, y la otra predominantemente heterogénea pero aún individualizada.

Si se retrocede en la evolución e itinerario de ambas profesiones, parece lógico que se bifurquen sus enfoques de enseñanza, ya que cada una emerge desde escenarios históricos, sociales, políticos y culturales distintos. La masificación de la enseñanza entre los años 50 y 60 , que fue privilegio de grupos de élite a cargo de eclesiásticos durante la época colonial y republicana, desenvuelve todo un proceso de profesionalización del personal docente de primaria, siempre bajo el amparo del Estado con un fin público, de suplir demandas que implicaban un alto índice de analfabetismo y acervo cultural precario de la población (Núñez Prieto, 2007).

La renovación progresiva de las políticas de educación especial en Chile, desde que se crea la primera escuela latinoamericana para niñas y niños sordos en el año 1852 (Godoy et al., 2004) demuestra la trayectoria que ha experimentado esta modalidad de enseñanza en el sistema educativo chileno. Como profesión en los años 60, vinculada en su comienzo al campo de la medicina y psicología, se desarrolla con el objeto de la rehabilitación, la corrección o curación del déficit (Godoy et al., 2004). Y a pesar de que la intención de aminorar la exclusión social del estudiantado en condición de discapacidad a través de su incorporación a la educación regular y aula común, y que hoy nos lleva a encontrar un alto porcentaje de establecimientos educacionales adscritos a los Programas de Integración Escolar (PIE), que consideran, además, otras condiciones particulares y extraordinarias de aprendizaje, irrumpen y se han validado otros nuevos modos de segregación, pero ahora al interior del aula (Infante, 2010). El estudiantado con alguna apariencia no habitual a nivel cognitivo, conductual, de lenguaje, sensorial asociado 
http://doi.org/10.15359/ree.25-1.23

o no a un descenso general de aprendizaje o discapacidad, desde su diagnóstico hasta su incorporación a tal estrategia educativa (MINEDUC, 2019), pasa a formar parte de los llamados grupos de estudiantes PIE.

El hecho de que se les categorice por razones imperiosas de índole económico (subvención especial) de cierta forma deriva en "un círculo regulatorio que más que abrir espacios de participación, los restringe" (Infante, 2010, p. 290), pues el currículo escolar, que posee definiciones claras y estandarizadas sobre qué y cómo debe ser implementado en un tiempo determinado se traduce a que la niñez que presenta NEE queda fuera de estas definiciones y mientras más se desprenda su necesidad educativa de los parámetros curriculares, menos posibilidades de experimentar tareas de aula compartidas. Entonces, un escenario pedagógico de cualquier escuela podría exponer a estudiantes en un mismo espacio físico realizando tareas de aprendizaje totalmente diferentes, lo que dista bastante del discurso inclusivo.

Al revisar el progreso y evolución del término inclusión y cómo esta conquista el medio educativo, es necesario remontarse al discurso del año 1948 y a la Declaración Universal de Derechos Humanos, en la que se hace referencia a la libertad e igualdad en dignidad y derechos que cada persona tiene al nacer (Saleh, 2005) y que uno de esos es la educación (Balieiro Lodi, 2014). Asociada todavía, de acuerdo con Ainscow (2017), a algo parecido a un tipo de atención de niños o niñas, con alguna discapacidad, es producto "de un acercamiento gradual desde la exclusión hacia la educación especial, haciendo hincapié en la integración, para luego arribar a la idea de la educación inclusiva" (p. 39).

Esta "efervescencia" que ha causado el concepto de inclusión en los diferentes sectores del quehacer internacional y nacional hace ya unos años, del cual se apropia nuestro sistema educacional, al mismo tiempo lo posiciona en el desafío de incorporar tal propósito a las salas de clases. Las escuelas de Chile, principalmente las públicas, y que son las que acogen a este estudiantado, se encuentran nuevamente ante un cambio estructural. Lo que comienza en la década de los 90, con el incentivo de políticas educativas y estrategias para la integración de estudiantes en esta condición extraordinaria a la escuela regular, se transforma gradualmente a través de los Decretos de Educación N. ${ }^{\circ} 490 / 90$ de 1990, y Supremos N. 01/98, N. ${ }^{\circ} 374 / 9$ y N. ${ }^{\circ}$ 170/09 en lo que actualmente rige los ya mencionados, PIE (MINEDUC, 2013).

Fundamentalmente, este último decreto vigente (como ya se ha expuesto), al circunscribir las medidas que establecen los protocolos de diagnóstico, también lo hace en relación con el acceso y apoyo compensatorio que se requiera en cada caso.

En tal proceso y a cambio de la entrega de recursos económicos que garantizan una subvención especial, para tales estudiantes, los centros se comprometen a adquirir una cultura escolar cada vez más consciente de las diferencias individuales de sus estudiantes y de cómo esto debiera generar respuestas pedagógicas eficientes, para circunstancias de aula diversa, que incluya a todos y todas por igual. 
http://doi.org/10.15359/ree.25-1.23

http://www.una.ac.cr/educare

educare@una.ac.cr

En esta transición, subyugada al principio inclusivo, es que se decreta y promulga la ley 20.845 el año 2015, Ilamada Ley de Inclusión Escolar. La ley "regula la admisión de los y las estudiantes, elimina el financiamiento compartido y prohíbe el lucro en establecimientos educacionales que reciben aportes del estado" (MINEDUC, 2018, p. 1).

Como parte de una reforma al sistema educacional, en lo que respecta a los tres puntos focales, se fijan modos para la transformación de la educación subvencionada a gratuita, la eliminación de dividendos económicos para los centros educativos y de la selección al ingresar a ellos. Todo esto con el fin de asegurar que la calidad educativa se convierta en un derecho (MINEDUC, 2018).

Los movimientos sociales y el tenor político que adquieren cada vez más las demandas populares se ven evidenciadas desde el 2006 en manifestaciones masivas de alta convocatoria como, por ejemplo, la revolución pingüina estudiantil o la marcha en contra del actual sistema de pensiones (AFP) en el año 2016. Una década de cambios en cierta forma irreversibles que, como describe Castillo Peña (2017), se ven revelados en una ciudadanía que sale a la calle a exigirlos. Temáticas como las colusiones de los grandes monopolios, la corrupción en las instituciones del Estado o cúpulas políticas y gubernamentales, la igualdad de género, el feminismo y tantas otras, son insumo suficiente para que la desaprobación y cuestionamiento a las jerarquías y organización social agilicen legislaciones que den respuesta a las exigencias de una ciudadanía que se involucra en discusiones y decisiones de las que, quizás, nunca fue parte.

Castillo Peña (2017), basado en un informe del Programa de Naciones Unidas para el Desarrollo (PNUD), presume que la sociedad chilena estaría transitando por un proceso de politización y, con respecto a este, en educación y durante las movilizaciones estudiantiles comienzan a insinuarse, en su discurso, términos confrontados, "la temprana utilización de códigos del tipo desigualdad/igualdad, exclusivo/inclusivo, segregado/integrado, pobreza/ riqueza, vale decir, atributos que connotan o refieren a la inequidad social" (p. 38), que se quedarían por mucho tiempo, en la conciencia colectiva. Un argumento que años más tarde sería el medio preciso o insumo suficiente para hacer notar la necesidad y casi urgencia de legislar sobre inclusión en Chile. Esta ley es parte de variadas medidas enmarcadas que suponen avances en la calidad de la educación principalmente pública y oportunidades de acceso a tal (Delpiano et al., 2017).

El asunto confluye en que existen eslabones de esta trama social que demuestran una insuficiencia en tales acciones que pretenden equidad y las mismas posibilidades para todos y todas. Las personas protagonistas, los niños y las niñas que no aprenden igual que el resto o la mayoría de sus compañeros y compañeras y, que en una clase de matemática se descuelgan de las tareas de rutina (Jimeno, 2006). Estos grupos que presentan, por diferentes razones, obstáculos en su aprendizaje, no podrían salir a la calle a exigir y están sujetos a la oferta educativa que el centro (la mayoría de las veces) de alta vulnerabilidad y docentes les brindan.

8 
http://doi.org/10.15359/ree.25-1.23

Hoy, se aspira a hallar consensos acerca de cómo se comporta en matemáticas un niño o una niña con dificultades y en qué debiera fundamentarse su reeducación. Para Duval (2016), la trascendencia de los elementos semióticos son fuente de la comprensión e incomprensión en matemáticas, la multiplicidad de sistemas de representación de un mismo objeto matemático propicia el acceso exclusivo a este. Estas barreras se manifiestan "ligadas al reconocimiento de una cohesión interna de cada sistema de representación y de otra externa entre ellos" (Barallobres, 2016, p. 52). Según Gónzalez et al. (2017), diversas disciplinas, entre ellas la didáctica de la matemática y la psicología cognitiva, concuerdan en el apremio de reparar en los componentes que forman parte de la construcción del conocimiento matemático, lo que comprende un análisis profundo de las problemáticas que se presentan en la etapa escolar, de cómo son evaluadas y mediadas a nivel pedagógico. La discusión o reflexión, entonces, giraría en torno a qué lineamientos son los adecuados, para propiciar el potencial matemático de este estudiantado que suele, además, subestimarse. Porque, se confrontan posturas que instalan al profesorado de educación especial o diferencial y al de matemática, en un intento de trabajo en colaboración, que se vuelve difuso e ineficaz, al ser llevado a las prácticas de aula, debido a proposiciones pedagógicas opuestas, fundadas en enfoques teóricos muy distintos. Una de ellas sugiere una instrucción explícita, directa y, la otra, la del aprendizaje matemático por indagación, diseño de estrategias y construcción de elementos conceptuales (Lambert, 2018).

La creencia de que el estudiantado con NEE en matemáticas no posee herramientas cognitivas para construir su propio conocimiento y que solo le aporta a su aprendizaje una enseñanza exigua y procedimental (Lambert, 2018) hace evidente que la planificación e implementación de la enseñanza para estos grupos, en el aula, es limitada.

Al compilar todos los antecedentes presentados, cabe al menos cuestionarse sobre qué está ocurriendo en las aulas chilenas para asegurar la igualdad y calidad de opciones de aprendizaje en matemática e indagar más profundamente en esta temática y qué contribución, desde sus fundamentos, puede entregar la didáctica de la matemática, para establecer directrices comunes de contención, entre los diferentes agentes que forman parte de la estructura educativa nacional que, de alguna manera, inciden en este fenómeno. Es solo hacerse cargo del futuro de individuos que, por derecho, deben poder incorporarse a las mismas oportunidades de adquisición del conocimiento matemático, independiente de sus historias, etnias, cultura, nivel social, motivaciones y características individuales de aprendizaje.

\section{Prácticas de enseñanza matemática y realidad pedagógica en espacios vulnerables: Reportes de una experiencia investigativa}

La etimología de la palabra diversidad, que expresa la cualidad de la abundancia y la variedad, proveniente del latín y significa girar en dirección opuesta. 
http://doi.org/10.15359/ree.25-1.23

http://www.una.ac.cr/educare

educare@una.ac.cr

"La noción de Diversidad origina amplias e indefinidas representaciones conceptuales" (Filippi, 2013, p. 9). Hacemos referencia a ella cuando intentamos definir la exuberancia de variados elementos en un contexto determinado como un término que, a su vez, adquiere diferentes connotaciones, dependiendo de cuál sea el motivo de aludir a él. Hoy, hablar de diversidad es habitual, hablar de derechos y segregación es una constante insignia de lucha para los grupos minoritarios, se recurre a ella como si llevara implícita la exigencia de ser respetada.

Sin embargo, esta condición, considerada excepcional, siempre ha existido, lo heterogéneo es natural y propio de los seres humanos. Es parte de un estado que precede inclusive al despliegue evolutivo de las especies. Desde una perspectiva biológica, es lo que propicia la evolución de la vida y, según la teoría darwiniana (Thompson, 2003), lo que permite la adaptación a condiciones adversas e inciertas del medio. Darwin incorpora, hace siglos, la idea de que las conmutaciones en la naturaleza y la cualidad de la desemejanza, permiten la estabilidad de los ecosistemas, "son las diferencias existentes entre los organismos de una especie las que, al magnificarse en el espacio y en el tiempo, producirán nuevas poblaciones, nuevas especies, y por extensión, toda la diversidad biológica" (Barbadilla, 1999, p. 606).

La variabilidad genética que tiende a la diferenciación de las especies se debe a la trascendencia de mutaciones aleatorias o intencionadas y fluctuaciones que originan la multiplicidad de organismos que viven, conviven e interactúan recíprocamente entre sí y con el ambiente. Esta diversidad, la biológica, aparece, entonces, como precursora del equilibrio en la naturaleza, lo que implica que los cambios y las transformaciones son tan fundamentales "que se puede inferir, que, aunque aparentemente ocurren al azar, responden a un continuo" (Filippi, 2013, p. 10).

Desde posturas paradigmáticas de la complejidad, lo estable en diferentes sistemas dinámicos u organizaciones no aparece asociado a la uniformidad, lo caótico es el principio del orden. $Y$ desde este enfoque, los procesos del universo y la naturaleza se comportan caóticamente de forma no lineal. Teorías que aceptan y validan la incertidumbre como parte de los fenómenos que nos rodean de toda índole y que según Campos Hernández (2008) a través de estas "se rompe con la búsqueda, casi esquizofrénica, del orden y el control propios del pensamiento occidental" (p. 3)

Diferentes ámbitos disciplinares e interdisciplinares se apropian, cada vez más, de esta inusual forma de entender el mundo y el comportamiento de los sucesos que parecen ser impredecibles.

De esta manera, la postmodernidad se deja seducir por una episteme de la indeterminación, la discontinuidad, el pluralismo y, claro está, opuesta a toda certeza. La incertidumbre aparece así, en la nueva realidad epistemológica, como una mediación necesaria entre el orden y el caos, resolviendo, finalmente, la discusión entre el ser y el devenir. (Campos Hernández, 2008, pp. 4-5) 
http://doi.org/10.15359/ree.25-1.23

Sin embargo y a pesar de que todo nos habla de que lo diverso e intermitente, lo azaroso y lo emergente es realidad, en tiempos de esta visible incerteza y de que las diferentes vertientes de las ciencias de la complejidad se han apropiado del quehacer científico moderno, contextos como el educativo aún reflejan esa visión determinista y homogeneizadora de cómo deben comportarse linealmente los procesos pedagógicos y de aprendizaje. En este afán de dominio y control, la diversidad, tal como lo afirma Filippi (2013), "es tratada como una condición caprichosa. Es lo que impide predecir resultados, lo que obstaculiza el logro de los mismos objetivos para un grupo de individuos" (p 15).

El trabajo investigativo realizado hace unos años, en una escuela pública urbana y periférica de la ciudad de Talca, en la que es reconocido un alto índice de vulnerabilidad debido a historiales socioculturales y económicos del alumnado que allí asiste, tales como migración, baja escolaridad y en muchos casos desatención de las familias, entrega información valiosa acerca de cuáles son los paradigmas educativos que imperan en contextos como el estudiado y qué creencias determinan el quehacer docente habitual en una sala de clases, de un establecimiento municipal en los que se concentran la mayoría de estudiantes con dificultades de aprendizaje, discapacidad intelectual y otras necesidades educativas especiales.

El objeto, sería explorar cómo se desarrollan las rutinas de aula, en entornos de notoria disparidad y cómo esta es abordada en las lecciones de matemática, con respecto a los referentes curriculares, legales y pedagógicos. La inquietud de aproximarse al suceso de estudio surge de antecedentes preliminares que muestran insuficiencias en el aprendizaje matemático escolar en el nivel básico y, por ende, bajos rendimientos en pruebas estandarizadas nacionales en esta disciplina, grandes brechas de resultados entre establecimientos públicos y privados, demandas educativas emergentes como la incorporación de estudiantes con NEE al aula común y procesos pedagógicos cada vez más exigentes y complejos en los que ya se vislumbraba, en ese tiempo, el apremio de una reestructuración en nuestro sistema educacional.

El caso estaba conformado por un grupo de treinta niños y niñas, que cursaban el $3^{\circ}$ grado de educación básica, su maestra de aula y diferencial de apoyo. En él, siete con diagnósticos con NEE y del resto varios sujetos evidenciaban descenso en el aprendizaje matemático y dificultades en la adquisición de la lectoescritura.

La intención de este trabajo llevaría a analizar los componentes didácticos en el aula de matemática en relación con su heterogeneidad, en el contexto de las necesidades educativas especiales. Y a través de diferentes mecanismos de indagación, es llevado a cabo el proceso que pronto comenzaría a dilucidar los diferentes cuestionamientos y conjeturas de la investigación, que permiten finalmente consolidar información trascendente y dar respuesta a su principal interrogante, con respecto a si el profesorado de matemática y profesorado especialista están preparados didáctica y metodológicamente para acudir a la diversidad en el aula y, si el sistema educativo provee las condiciones para tal propósito. Los soportes teóricos y bibliográficos, durante el transcurso del 
http://doi.org/10.15359/ree.25-1.23

http://www.una.ac.cr/educare

educare@una.ac.cr

estudio moldearon, tanto los constructos teórico-conceptuales como los tópicos y criterios de análisis de los datos recopilados a través de entrevistas semiestructuradas a ambas docentes y observación directa de clases. La categorización apriorística permitiría organizar las nociones en dos principales agrupaciones conceptuales: aprendizaje matemático y diversidad de aula.

Desde ahí se desprenden argumentos operacionalizados en las categorías: sistemáticas de enseñanza, estrategias de enseñanza, estrategias de aprendizaje, diversidad de aula y aula inclusiva. Aparatos conceptuales que decodificados e interpretados desde la óptica del enfoque ontosemiótico (EOS) (Diaz Godino et al., 2006) y los criterios de idoneidad didáctica son abordados por la dimensiones epistémica, cognitiva, interaccional y afectiva.

La epistémica y cognitiva involucran los modelos de enseñanza, la gestión de tareas matemáticas y su pertinencia, referida a los "significados implementados y pretendidos" (Diaz Godino et al., 2006, p. 7), el nivel de concordancia de estos significados con los de cada estudiante en una relación coherente con las capacidades potenciales y habilidades matemáticas, la exigencia cognitiva en los tipos de problemas y la aplicación de los conocimientos para resolverlos. La interaccionalaborda los conflictos en la interacción entre docente y estudiantes, y entre estudiantes; las discusiones, conjeturas, intercambio de ideas, espacios de dialogo reflexivo sin estereotipos. La afectiva trata el grado de compromiso hacia el alumnado durante el proceso, al propiciar el debate y la autonomía, considerando para este propósito sus intereses, razonamientos, puntos de vista, trayectorias de aprendizaje, actitudes y necesidades, para fomentar la participación e inclusión en el espacio matemático de aula (Godino et al., en Breda et al., 2018).

Los principales hallazgos del trabajo realizado dan cuenta de que, en la realidad y caso estudiado, el escenario pedagógico al que se enfrentan ambas profesoras, una de ellas cotidianamente, se despliega entre marcados contrastes y notorias diferencias de aprendizaje. Coincidente con lo señalado por Jimeno (2006), acerca de que "unos cuantos estudiantes captan rápidamente los conceptos y avanzan sin ningún tipo de problemas, otros tienen un ritmo muy lento, aunque no tengan dificultades específicas y unos pocos muestran serias dificultades en algunos aspectos del aprendizaje matemático" (p. 1).

En los episodios de clase y discurso docente, se aprecian barreras en relación con aspectos metodológicos y formalidad didáctica de la disciplina, para propiciar la reflexión, trabajo en equipo, desarrollo de habilidades cognitivas o metacognitivas y de pensamiento inductivo en el estudiantado. Las dinámicas de aula son reguladas a través de una relación vertical entre profesora de aula y estudiantes, con un rol adjunto de la educadora diferencial, recurriendo a clases de tipo magistral y expositivas con una marcada orientación conductista. Al respecto, indica Orton (1996), es interesante considerar si aún en la enseñanza de la matemática son aceptadas ciertas acciones que responden a esta tendencia, que convierte la implementación de contenidos curriculares en el fin del proceso, sin la clara programación de promover habilidades cognitivas específicas hacia la homogenización del proceso de enseñanza y aprendizaje matemático. 
http://doi.org/10.15359/ree.25-1.23

Tanto la planeación e implementación de las actividades matemáticas son pensadas para el estudiantado con niveles y ritmos de aprendizaje esperados, en las que se priorizan alcances y propósitos consistentes con el dominio procedimental de la disciplina, de mecanismos y ejercitación por sobre el trabajo matemático autónomo, en el que la resolución de problemas esta casi ausente en oposición a una enseñanza matemática que enfatiza en los hechos metacognitivos, fructuosos al desarrollar en el estudiantado un sentido de sí mismo como agente cognitivo (Favell, 1987, citado en Clements, 1999).

El modelo de atención a la diversidad, ya sea por diferentes factores, aparece lejano, desde las falencias en la implementación de las normativas vigentes, que rigen los PIE, hasta las prácticas pedagógicas un tanto inconsistentes, que debido a las creencias de ambas y a las condiciones del medio en el que se desarrollan, propician la exclusión más que la inclusión, y la segregación del estudiantado en el aula de matemática. La realidad cotidiana en circunstancias pedagógicas, con una marcada desemejanza de aprendizajes, en la clase de matemática, como las del caso estudiado, diverge del ideal platónico de una educación inclusiva, pues una respuesta adecuada para el estudiantado con descenso general de aprendizaje y dificultades específicas consiste en una serie de acciones planificadas e implementadas en colaboración, diversificación de estrategias y recursos didácticos (MINEDUC, 2019) y procedimientos evaluativos coherentes.

Con base en las evidencias y resultados reportados del proceso investigativo se sugieren ciertas recomendaciones y aspectos que podrían proyectar para entornos pedagógicos en condiciones similares, este salto necesario hacia una verdadera educación inclusiva en la clase de matemática.

- El trabajo matemático de aula. Debiera centrarse en la resolución de problemas en contextos auténticos y cercanos a la realidad del estudiantado con el propósito de desarrollar la comprensión y una estructura conceptual que les permita aprender (Aravena, 2007) y, desde el punto de vista epistémico, en la secuencia de tareas considerar actividades matemáticas de relevancia, la utilización de modos de expresión verbal, gráfica, simbólica o analítica y el tránsito entre las mismas para la observación de fenómenos desde diversas perspectivas.

- Modelos de enseñanza para atender a la diversidad. Este propósito en una clase de matemática implica la búsqueda de teorías y modelos que permitan reinterpretar y reelaborar el trabajo de aula (Jorba y Sanmartí, 1996), que orienten la enseñanza y organización de las clases, con el fin de responder a las características estudiantiles, anticiparse a sus ideas, posibles dificultades y obstáculos para que el aprendizaje de conceptos y métodos sea significativo (Aravena et al., 2011).

- Metodologías de enseñanza matemática. Se requiere preparar a docentes en modelos de transposición didáctica (Brousseau, 1998), métodos, recursos de enseñanza y 
http://doi.org/10.15359/ree.25-1.23

http://www.una.ac.cr/educare

educare@una.ac.cr

estrategias evaluativas enfocados al desarrollo de habilidades para todos los niños y todas las niñas, a generar espacios de diálogo compartido, el entendimiento de las ideas, conceptos y procesos matemáticos.

- Formación del profesorado. Es necesario formar desde las prácticas tempranas en experiencias inclusivas y el diseño e implementación de secuencias de aprendizaje que integren aspectos psicopedagógicos, didácticos y evaluativos para atender a la diversidad en el aula. Esto le permitiría codificar ambas perspectivas (teórica y práctica) a modo de retroalimentación, respecto de creencias, estereotipos u otras manifestaciones inconscientes y estar preparado para enseñar a niños y niñas de diferentes realidades, contextos o culturas.

- El modelo escolar. Reconocer la diversidad y acudir a ella representa, para la organización escolar, acciones como la de adaptar su modelo educativo, incorporar equipos multidisciplinarios y el desarrollo de un currículo equitativo (Diaz Godino, 2005), cuya gestión y concreción considere el aprendizaje como un componente social más, de participación con los mismos derechos y las mismas oportunidades (D'Ambrósio, 2005).

- Diversidad de aprendizajes matemáticos. Sería conveniente incorporar dinámicas de clases y experiencias de enseñanza compartidas que, en la visión de la idoneidad afectiva, favorecen el compromiso del personal docente de aula y el diferencial con todo el alumnado, la priorización de objetivos de aprendizaje comunes, la utilización de formas variadas de representación del conocimiento matemático y de materiales didácticos adaptados y concretos que, de acuerdo con Crisol Moya et al. (2015), facilitan la labor educativa, la hacen versátil y extienden las oportunidades de aprendizaje.

\section{Consideraciones finales}

Al mirar lo expuesto en el texto y comparar ambas secciones se pueden apreciar puntos en los cuales existen evidentes coincidencias en cuanto a los nudos críticos abordados, a pesar de que se describen antecedentes de épocas con una diferencia de al menos cinco o seis años. Esto significa que el avance hacia la mejora en la oferta educativa, para el estudiantado con necesidades educativas especiales, ha sido, al menos, lento.

Hoy en día, las exigencias de una educación para la diversidad y las pretensiones de los parámetros inclusivos -bajo el alero de la igualdad de derechos y equidad social-impuestos a nivel de diferentes esferas del quehacer nacional, sitúan al sistema educacional chileno en un dilema que, quizás, solo vemos en profundidad, quienes hemos sido parte de la realidad en las aulas públicas, al protagonizar la enseñanza en contextos pedagógicos calificados de desempeño difícil. 
http://doi.org/10.15359/ree.25-1.23

Para quienes hemos mirado la trayectoria de este fenómeno, desde adentro y desde afuera, como docentes de aula y como docentes que forman el nuevo profesorado, el desafío está en: ¿cómo abordar este proceso ya irreversible?, de inclusión de aquellos niños y niñas que no siempre reciben lo que requieren para alcanzar las mismas oportunidades que el resto de sus compañeros y compañeras de su misma edad y nivel.

Se hace urgente examinar, desde cada estamento en la estructura de nuestro sistema educativo, cómo las entidades ministeriales, la formación inicial docente, la docencia en ejercicio, las comunidades escolares, qué es lo que nos falta para entregar lo que hemos prometido a los padres, las madres, a los niños y las niñas, a la sociedad, para lograr esa educación de calidad, equitativa y justa, que tanto se anhela.

Pues no basta solo con incorporar estudiantes con alguna necesidad de aprendizaje excepcional al aula común y pensar que esto es gran parte de la estrategia inclusiva.

Hablar de inclusión en educación es asumir estos cambios, dejar los formatos rígidos y flexibilizar el currículo, reparar en los procesos y utilizar los resultados para redireccionar estrategias. Es propiciar las condiciones desde las esferas más altas, para una transformación en las prácticas pedagógicas factibles y el desarrollo previo de competencias comunes en formación inicial del profesorado en didácticas inclusivas, interdisciplinariedad, codocencia y trabajo colaborativo.

La enseñanza de la matemática por estos días debe dirigirse al aprendizaje de todo el estudiantado en el aula y no es efectiva, si favorece solo a alguna parte, ya que no estaría respondiendo al principio fundamental de una educación matemática para la diversidad.

\section{Declaración de Material complementario}

Este artículo tiene disponible, como material complementario:

-La versión preprint del artículo en https://doi.org/10.5281/zenodo.3661190

\section{Referencias}

Aguilar Villagrán, M., Aragón Mendizábal, E. y Navarro Guzmán, J. I. (2015). Las dificultades de aprendizaje de las matemáticas (DAM). Estado del arte. Revista de Psicología y Educación, $10(2), 13-42$.

Ainscow, M. (2017). Haciendo que las escuelas sean más inclusivas: Lecciones a partir del análisis de la investigación internacional. Revista de Educación Inclusiva, 5(1), 39-49 https://dialnet. unirioja.es/servlet/articulo?codigo $=4105297$ 
http://doi.org/10.15359/ree.25-1.23

http://www.una.ac.cr/educare

educare@una.ac.cr

Aravena, M. (2007). Método de resolución de problemas. Lesson Study de Japón. ¿Es posible una aproximación a la realidad chilena? Actas XXI Jornadas de Matemática de la Zona Sur (pp. 60). Concepción, Chile.

Aravena, M., Caamaño, C., González, J., Cabezas, C. y Córdova, F. (2011). Resolución de problemas en contextos de aplicación. Propuesta metodológica en la formación inicial de profesores de matemática. Tabor.

Balieiro Lodi, A. (2014). Declaraciones de la UNESCO, educación de sordos y educación inclusiva: Un analisis de la politica nacional de educación de Brasil. Psicología, Conocimiento y Sociedad, 4(2), 261-294. https://revista.psico.edu.uy/index.php/revpsicologia/article/ view/213/216

Barallobres, G. (2016). Diferentes interpretaciones de las dificultades de aprendizaje en matemática. Educación matemática, 28(1), 39-68. https://doi.org/10.24844/EM2801.02

Barbadilla, A. (1999). La selección natural: "Me replico luego existo". Boletín de la S.E.A., 26, 605612. http://sea-entomologia.org/PDF/BOLETIN 26/B26-041-605.pdf

Breda, A., Font Moll, V., do Rosário Lima, V. M.y Villela Pereira, M. (2018). Componentes e indicadores de los criterios de idoneidad didáctica desde la perspectiva del enfoque ontosemiótico Transformación, 14(2), 162-176. http://scielo.sld.cu/pdf/trf/v14n2/trf03218.pdf

Brousseau, G. (1998). Théorie des situations didactiques. La Pensée Sauvage

Campos Hernández, R. (2008). Incertidumbre y complejidad: Reflexiones acerca de los retos y dilemas de la pedagogía contemporánea. Actualidades Investigativas en Educación, 8(1), 1-13. https://revistas.ucr.ac.cr/index.php/aie/article/view/9326

Castillo Peña, J. (2017). El proceso de politización como contexto de la ley de inclusión escolar. En Ministerio de Educación de Chile (Ed.), El primer gran debate de la reforma educacional: Ley de inclusió escolar (pp. 26-47). Ministerio de Educación de Chile. https://www.mineduc. cl/wp-content/uploads/sites/19/2018/03/libro Inclusio\%CC\%81n final.pdf

Clements, M. A. (1999). Planteamiento y resolución de problemas: ¿Es relevante Polya para las matemáticas escolares del siglo XXI? Suma, 30, 27-36. https://revistasuma.es/revistas/30febrero-1999/planteamiento-y-resolucion-de.html

Crisol Moya, E., Martínez Moya, J. y El Homrari, M. (2015). El aula inclusiva. Condiciones didácticas y organizativas. Revista Nacional e Internacional de Educación Inclusiva, 8(3), 254-270. https://revistaeducacioninclusiva.es/index.php/REl/article/view/100

D’Ambrósio, U. (2005). Sociedade, cultura, matemática e seu ensino. Educação e Pesquisa, 31(1), 99-120. 
http://doi.org/10.15359/ree.25-1.23

http://www.una.ac.cr/educare educare@una.ac.cr

Delpiano A., Rucks del Bó, S. y Perez Gúñez, A. (2017). Presentaciones. En Ministerio de Educación de Chile (Ed.), El primer gran debate de la reforma educacional: Ley de inclusió escolar (pp. 8-11). Ministeiro de Educación de Chile. https://www.mineduc.cl/wp-content/uploads/ sites/19/2018/03/libro Inclusio\%CC\%81n final.pdf

Diaz Godino, J. (2005). Presente y futuro de la investigación en didáctica de las matemáticas. GT: Educação Matemática, 19, 1-24. http://www.ufrrj.br/emanped/paginas/conteudo producoes/docs 29/presente.pdf

Diaz Godino, J., Bencomo, D., Font, V. y Wilhelmi, M. R. (2006). Análisis y valoración de la idoneidad didáctica de procesos de estudio de las matemáticas. Paradigma, 27(2), 221-252. https:// www.ugr.es/ jgodino/funciones-semioticas/idoneidad-didactica.pdf

Duval, R. (2016). Un análisis cognitivo de problemas de comprensión en el aprendizaje de las matemáticas. En R. Duval y A. Sáenz-Ludlow (Autores), Comprensión y aprendizaje en matemáticas: Perspectivas semióticas seleccionadas (pp. 61-94). Universidad Distrital Francisco José de Caldas. https://www.academia.edu/27318074/Comprensi\%C3\%B3n y aprendizaje en matem\%C3\%A1ticas perspectivas semi\%C3\%B3ticas seleccionadas

Echeita Sarrionandía, G., y Ainscow, M. (2011). La educación inclusiva como derecho. Marco de referencia y pautas de acción para el desarrollo de una revolución pendiente. Tejuelo, 12, 26-46. https://repositorio.uam.es/bitstream/handle/10486/661330/educacion echeita TEJUELO 2011.pdf?sequence=1

Filippi, C. (2013). Enseñar en la diversidad: Didáctica e inclusión en el aula de matemática [Tesis de maestría inédita]. Universidad Católica del Maule, Chile.

Godoy, M. P., Meza M. L. y Salazar, A. (2004). Antecedentes históricos, presente y futuro de la educación especial en Chile. Ministerio de Educación, Programa de Educación Especial. https://bibliotecadigital.mineduc.cl/bitstream/handle/20.500.12365/610/MONO-523. pdf? sequence $=1$

González, I., Benvenuto, G. y Lanciano, N. (2017). Dificultades de aprendizaje en matemática en los niveles iniciales: Investigación y formación en la escuela italiana. Psychology, Society \& Education, 9(1), 135-145. https://doi.org/10.25115/psye.v9i1.468

Hécaen, H., Angelergues, R. y Houillier, S. (1961). Les variétés cliniques des acalculies au cours des lésions rétrorolandiques: Approche statistique du problème. Revue neurologique, 105, 85-103.

Infante, M. (2010). Desafíos a la formación docente: Inclusión educativa. Estudios pedagógicos, 36(1), 287-297. https://doi.org/10.4067/S0718-07052010000100016 
http://doi.org/10.15359/ree.25-1.23

http://www.una.ac.cr/educare

educare@una.ac.cr

Jimeno, M. (2006). ¿Por qué las niñas y los niños no aprenden matemáticas? Octaedro.

Jorba, J. y Sanmartí, N. (1996). Enseñar, aprender y evaluar: Un proceso de evaluación contínua. Propuesta didáctica para las ciencias de la naturaleza y matemática. Ministerio de Educación y Cultura. https://www.researchgate.net/publication/39143780 Ensenar aprender_y evaluar_un proceso de regulacion_continua propuestas didacticas para_las areas de Ciencias de la Naturaleza y Matematicas

Kaufmann, L., Mazzocco, M. M., Dowker, A., von Aster, M., Göbel, S. M., Grabner, R. H., Henik, A., Jordan, N. C., Karmiloff-Smith, A. D., Kucian, K., Rubinsten, O., Szucs, D., Shalev, R. y Nuerk, H.-C. (2013). Dyscalculia from a developmental and differential perspective. Frontiers in psychology, 4, 1-5. https://doi.org/10.3389/fpsyg.2013.00516

Lambert, R. (2018). "Indefensible, illogical, and unsupported"; countering deficit mythologies about the potential of students with learning disabilities in mathematics. Education Sciences, 8(2), 1-12. https://doi.org/10.3390/educsci8020072

Lombardino, M. (2017). Trastorno o dificultad en la identificación temprana de las dificultades de aprendizaje [Tesis doctoral]. Universidad de Extremadura España. http://dehesa.unex. es/static/flexpaper/template.html?path=/bitstream/handle/10662/6550/TDUEX 2017 Lombardino M.pdf?sequence=1\&isAllowed=y\#page=1

Maher, C. A., Sigley, R., Sullivan, P. y Wilkinson, L. (2018). An international perspective on knowledge in teaching mathematics. The Journal of Mathematical Behavior, 51, 71-79. https://doi.org/10.1016/j.jmathb.2018.05.002

Ministerio de Educación de Chile. (2010). División jurídica. Decreto con toma de razón N. 0170 Santiago -14/05/2009. Fija normas para determinar los alumnos con necesidades educativas especiales que serán beneficiarios de las subvenciones para educación especial. Ministerio de Educación de Chile. http://especial.mineduc.cl/wp-content/ uploads/sites/31/2016/08/201304231500550.DEC200900170.pdf

Ministerio de Educación de Chile. (2011). Estándares orientadores para egresados de carreras de pedagogía en educación básica. Estándares pedagógicos y disciplinarios (2. ${ }^{a}$ ed.). Autor. https://www.cpeip.cl/wp-content/uploads/2019/03/Est\%C3\%A1ndares B\%C3\%A1sica.pdf

Ministerio de Educación de Chile. (2013). Análisis de la implementación de los programas de integración escolar (PIE) en establecimientos que han incorporado estudiantes con necesidades educativas especiales transitorias (NEET). https://especial.mineduc.cl/wp-content/uploads/ sites/31/2016/08/Resumen Estudio ImplementacionPIE 2013.pdf

Ministerio de Educación de Chile. (2018). Ley de inclusión escolar. https://liderazgoescolar. mineduc.cl/ley-inclusion-escolar-2/ 
http://doi.org/10.15359/ree.25-1.23

Ministerio de Educación de Chile. (2019). Programa de Integración escolar PIE. Manual de apoyo a la inclusión escolar. Autor. https://especial.mineduc.cl/gestion-operacional-pie/ documentos-apoyo-ingreso-pie/

Núñez Prieto, I. (2007). La profesión docente en Chile: Saberes e identidades en su historia. Revista Pensamiento Educativo, 41(2), 149-164.

Orton, A. (1996). Didáctica de las matemáticas. Morata.

Programa de las Naciones Unidas para el Desarrollo. (2015). Desarrollo humano en Chile. Los tiempos de la politización 2015. Los tiempos de la politización. Autor. https://www. cl.undp.org/content/chile/es/home/library/human development/los-tiempos-de-lapolitizacion.html

Saleh, L. (2005). Inclusión de personas con discapacidad: Una mirada internacional y nacional. En C. Bañados (Ed.), Seminario internacional: Inclusión social, discapacidad y políticas públicas (pp. 9-26). Ministerio de Educación; MINSAL; UNESCO; Ministerio del Trabajo; PNUD; FONADIS y OIT. http://www.lacusaragon.org/seminario-internacional-inclusionsocial-discapacidad-y-politicas-publicas-chile-2005-un-documento-muy-actual/

Tenorio, S. (2011). Formación inicial docente y necesidades educativas especiales. Estudios pedagógicos, 37(2), 249-265. https://dx.doi.org/10.4067/S0718-07052011000200015

Thompson, J. N. (2003). El proceso coevolutivo. FCE.

United Nations Eductional, Scientificand Cultural Organization. (2005). Guidelines for inclusion: Ensuring access to education for all. UNESCO. https://unesdoc.unesco.org/ark:/48223/ pf0000140224

Winter, E. y O'Raw, P. (2010). Literature review of the principles and practices relating to inclusive education for children with special educational needs. National Council for Special Education. http://www.nabmse.org/wp/wp-content/uploads/downloads/2012/07/NCSE Inclusion.pdf 